\title{
White coat hyperglycaemia: disparity between diabetes clinic and home blood glucose concentrations
}

\author{
Lesley V Campbell, Sheena M Ashwell, Mark Borkman, Donald J Chisholm
}

\begin{abstract}
Objectives-To identify patients with discrepantly high clinic blood glucose concentrations compared with self reported values and to assess whether such patients have errors in self monitoring technique. To determine whether, in patients with good technique, the discrepancy is a transient phenomenon related to clinic attendance.
\end{abstract}

Design-Prospective study of diabetes clinic patients recruited over six months.

Setting-Outpatient diabetes clinic of a teaching hospital.

Subjects-34 consecutive patients with noninsulin dependent diabetes who had had at least two consecutive clinic blood glucose concentrations more than $5 \mathrm{mmol} / \mathrm{l}$ higher than the mean self reported concentration.

Main outcome measures-Assessment of monitoring technique; presence of cognitive or physical impairment; serum fructosamine concentration; home and clinic blood glucose concentrations.

Results -15 of 34 patients had errors in monitoring technique, 12 of whom had cognitive or physical impairment. In the remaining 19 , the mean (SD) blood glucose concentrations of capillary and venous samples taken at home $(10 \cdot 2(0 \cdot 6)$ and 12.2 $(1 \cdot 1) \mathrm{mmol} / \mathrm{l}$ respectively) were significantly lower than in those taken at the clinic $(16.8(1.6) \mathrm{mmol} / \mathrm{l}$, $p<0.0002)$. The fructosamine concentration was significantly higher in patients with monitoring errors than those without $(2.4(0.4) \quad v \quad 1.8(0.4)$ $\mathrm{mmol} / \mathrm{l}, \mathrm{p}<0 \cdot 0001$ ).

Conclusions-"White coat" hyperglycaemia was detected in about half the patients but errors in technique accounted for the rest of the discrepancies. Patients' ability should be assessed before teaching self monitoring and the technique checked regularly.

Garvan Institute of Research, St Vincent's Hospital, Sydney, Australia Mark Borkman, research fellow

Donald J Chisholm, professor of endocrinology

Correspondence to: Dr Campbell.

BMF 1992;305:1194-6

\section{Introduction}

Self monitoring of blood glucose concentration is commonly used to assess glycaemic control in diabetes, and when done properly it is accepted as a reliable and accurate indicator.' A survey of our diabetic outpatient clinic showed that $97 \%$ of insulin dependent and $67 \%$ of non-insulin dependent diabetic subjects were

TABLE I-Clinical characteristics and glycaemic measures of the error and non-error groups. Concurrent data from 412 non-insulin dependent diabetic patients attending the clinic over the same six months.Values are means with SD in parentheses

\begin{tabular}{|c|c|c|c|c|}
\hline & $\begin{array}{c}\text { All subjects } \dagger \\
(\mathrm{n}=34)\end{array}$ & $\begin{array}{l}\text { Error group } \\
\quad(\mathrm{n}=15)\end{array}$ & $\begin{array}{l}\text { Non-error } \\
\text { group } \\
(\mathrm{n}=19)\end{array}$ & $\begin{array}{l}\text { All non-insulin } \\
\text { dependent diabetic } \\
\text { patients at clinic } \\
(n=412)\end{array}$ \\
\hline Age (years) & $68(8 \cdot 8)$ & $68(10 \cdot 8)$ & $68(7 \cdot 1)$ & $65(10 \cdot 1)$ \\
\hline Duration of diabetes (years) & $8(9)$ & $8(7)$ & $9(5)$ & \\
\hline \multicolumn{5}{|l|}{ Blood glucose (mmol/li) } \\
\hline Self monitoring & $9 \cdot 1(1 \cdot 8)$ & $10 \cdot 1(2 \cdot 0)$ & $8 \cdot 4(1 \cdot 1)^{\star \star}$ & \\
\hline Clinic & $17 \cdot 7(2 \cdot 5)$ & $18.9(2.9)$ & $16 \cdot 8(1 \cdot 6)^{\star}$ & $11 \cdot 3(4 \cdot 1)$ \\
\hline $\begin{array}{l}\text { Difference between clinic and self } \\
\text { monitoring }\end{array}$ & $8 \cdot 6(1 \cdot 8)$ & $8 \cdot 8(2 \cdot 1)$ & $8 \cdot 4(1 \cdot 5)$ & \\
\hline Clinic serum fructosamine $\ddagger(\mathrm{mmol} / \mathrm{l})$ & $2 \cdot 0(0 \cdot 6)$ & $2 \cdot 4(0 \cdot 4)$ & $1 \cdot 8(0 \cdot 4)^{\star \star}$ & $1.8(0.4)$ \\
\hline
\end{tabular}

monitoring their own glucose concentrations..$^{2}$ In our experience elderly patients with diabetes can be taught monitoring; routine checking of technique within two weeks has shown performance comparable with that in younger patients.

In the outpatient clinic doctors use the patient's clinic blood glucose concentration and the self recorded $\log$ book to assess recent glycaemic control. It is not uncommon to find that the patient's clinic blood glucose concentration is high compared with the self reported levels. Patients often ascribe this to the stresses of attending clinic such as transport, parking, erratic eating, waiting, and fear of the clinic procedures (or staff). This is analogous to the phenomenon of "white coat hypertension," ${ }^{3}$ in which blood pressure is higher in the clinic than at home. In some patients the glucose discrepancy is large enough to warrant change of treatment if the clinic test were the true reflection of glycaemic control. This presents the clinician with a management dilemma. A large discrepancy which is present at consecutive clinic visits suggests either inaccurate self monitoring or transient hyperglycaemia at clinic which is unrepresentative of usual glycaemic control. Although indices of long term control are also measured, such as glycated haemoglobin or serum fructosamine concentration (a measure of the glycation of albumin), the results are not generally available while the patient is at the clinic. Uncertainty about a patient's current glycaemic control can lead doctors to adjust treatment inappropriately. Some clarification of the likely causes of the discrepancy would be beneficial in managing such patients.

In this study we set out, firstly, to identify those patients with a repeated and large glycaemic discrepancy; secondly, to determine whether such discrepancy was related to errors in self monitoring technique; and, thirdly, if there was no technique error to examine if the discrepancy was due to transien hyperglycaemia related to clinic attendance.

\section{Subjects and methods}

SUBJECTS

Participants were recruited over six months from patients attending the weekly outpatient diabetes clinic of our hospital, an inner city teaching hospital. Of the 412 patients with non-insulin dependent diabetes mellitus who attended the clinic during this period 283 were monitoring their blood glucose concentration at home. Irrespective of treatment, these patients were eligible for the study if, at two consecutive clinic visits, the clinic venous blood glucose concentration exceeded the mean of the previous month's self monitoring results by more than $5 \mathrm{mmol} / \mathrm{l}$. Patients with noninsulin dependent diabetes were studied as such patients are less likely to show random fluctuations in blood glucose concentration than those with insulin dependent diabetes ${ }^{4}$ and thus blood glucose profiles are more reproducible on a day to day basis.

The 34 consecutive patients satisfying these criteria all consented to take part in the study, which was approved by the hospital research and ethics committee. Table I shows their clinical characteristics and 
measures of glycaemic control. Data from the total non-insulin dependent diabetic population attending during the six months are also included to illustrate the nature of the clinic population. Our diabetes clinic is a referral clinic with an active policy of giving insulin to patients in whom tablets have failed, even if elderly: 125 of the $412(30 \%)$ non-insulin dependent patients attending the clinic were receiving insulin. The 34 patients with a glycaemic discrepancy had more severe diabetes: 21 were receiving insulin, the remainder oral treatment. Twenty nine had been taught monitoring at our diabetes centre. Fifteen patients had been monitoring for over three years, eight for one to three years, and the remainder for less than a year. Seventeen assessed glucose test strips only visually; the remainder used reflectance meters to read the strips.

\section{METHODS}

The figure shows the flow plan of the study. On the day of recruitment each patient was reviewed by a single experienced diabetes educator to identify errors in the technique of blood glucose monitoring together with any physical or cognitive defect which could contribute to such errors-for example, tremor, visual defects, difficulty in problem solving, or memory impairment. The presence of any emotional disorder of

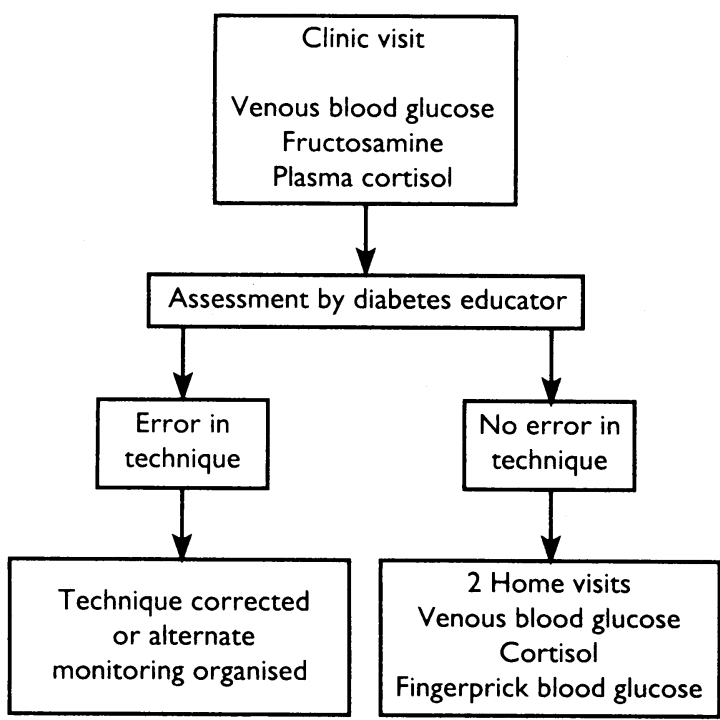

Flow plan for assessment of patients whose clinic blood glucose concentration was over $5 \mathrm{mmoll}$ higher than the average recorded value

sufficient degree to be mentioned in the patient's records was noted. Suitable strategies were implemented in those patients in whom errors in monitoring technique were identified (designated the error group). In patients with no detectable error (designated non-error group) glycaemic control was assessed in the home environment. The educator visited each patient's home twice, at a similar time of day and at the same time in relation to breakfast as the clinic venous blood glucose measurement. In random order capillary fingerprick and venous blood samples were collected to measure blood glucose concentration. Plasma cortisol concentration was also measured in the clinic and home venous blood samples, as a crude indicator of stress experienced by the patient. ${ }^{5}$

\section{BIOCHEMICAL ANALYSES}

The glucose concentrations in venous and capillary whole blood samples were measured with glucose oxidase impregnated wipe off strips (BM-Test-BG 20/800, Boehringer Mannheim, Germany) and a Reflolux 2 reflectance meter (Boehringer Mannheim, Germany). All venous blood glucose measurements were confirmed by a second analysis on the remaining
TABLE III-Mean (SD) blood glucose and plasma cortisol concentrations in non-insulin dependent diabetic patients with no error in home monitoring technique

\begin{tabular}{lll}
\hline & $\begin{array}{c}\text { Blood glucose } \\
(\mathrm{mmol} / \mathrm{l}) \\
(\mathrm{n}=19)\end{array}$ & $\begin{array}{c}\text { Plasma cortisol } \\
(\mathrm{nmol} / \mathrm{l}) \\
(\mathrm{n}=13)\end{array}$ \\
\hline $\begin{array}{l}\text { Clinic: } \\
\text { Venous whole blood }\end{array}$ & $16 \cdot 8(1 \cdot 6)$ & $418(112)$ \\
$\begin{array}{l}\text { Home: } \\
\text { Venous whole blood } \\
\text { Capillary whole blood }\end{array}$ & $\begin{array}{l}12 \cdot 2(1 \cdot 1)^{\star} \\
10 \cdot 2(0 \cdot 6)^{\star}\end{array}$ & $359(83) \dagger$ \\
$\begin{array}{l}\text { \# } \mathrm{p}<0 \cdot 001 \text { Compared with clinic value. } \\
+\mathrm{p}=0 \cdot 055 \text { Compared with clinic value; standard error of the mean } \\
\text { difference }=34 \text { nmol/l. }\end{array}$
\end{tabular}

sample with a glucose oxidase method (YSI 23 AM, Yellow Springs Instruments, Yellow Springs, Ohio). Plasma cortisol concentration was determined by radioimmunoassay. ${ }^{6}$ Serum fructosamine was measured by colorimetric reaction with nitroblue tetrazolium on an automated autoanalyser.

\section{STATISTICAL ANALYSES}

Statistical analyses were done with the Statview $512+$ statistical package (Abacus Concepts/Brainpower, Callabassas, California). Comparisons between the error and non-error groups for continuous variables were made by Student's $t$ test for unpaired data; the Wilcoxon rank signed test was used to compare the duration of diabetes and the magnitude of the discrepancy between clinic and self monitoring results. The $\chi^{2}$ test with Yates's correction or Fisher's exact test, when the numbers in the cells were small, was used for categorical variables. Student's $t$ test for paired data was used to compare clinic and home variables in the non-error group. Results are expressed as mean (SD) unless otherwise stated. Statistical significance was set at $\mathrm{p}<0.05$.

\section{Results}

Thirty four patients were eligible for the study because they had a large and repeated discrepancy between self monitoring and clinic blood glucose measurements. The mean (SD) clinic venous blood glucose concentration was $17 \cdot 7(2 \cdot 5) \mathrm{mmol} / \mathrm{l}$ with a discrepancy of $8.6(1.8) \mathrm{mmol} / \mathrm{l}$ (table I). The educator found that 15 patients (error group) had easily detected errors in monitoring technique that were of a type and sufficient severity to account for the glycaemic discrepancy. Table II records the frequency of the main errors. Visual assessment of test strips was more common in the error group than the non-error group $(11 / 15 v 6 / 19 ; p=0.037$, Fisher's exact test). Twelve of the 15 patients in the error group had a physical or cognitive disability compared with five of 19 in the non-error group ( $p=0 \cdot 005$, Fisher's exact test). Age, duration of diabetes, size of glycaemic discrepancy, treatment, and duration of self monitoring did not differ significantly in the two groups.

Table III gives the data from the 19 patients (nonerror group) studied at home. The mean (SD) blood glucose concentrations in capillary and venous samples collected at home $(10.2(0.6)$ and $12.2(1 \cdot 1) \mathrm{mmol} / \mathrm{l}$ respectively) were significantly lower than the clinic venous blood glucose concentration (16.8 (1.6) $\mathrm{mmol} / \mathrm{l}, \mathrm{p}<0.0002)$. Blood samples from both the clinic and home visits were available for measurement of cortisol in 13 patients; the concentrations were not significantly different (mean (SE) difference 59 (34) nmol; table III). No serious emotional disorder was recorded in any of the patients' records in either group.

The error group was significantly more hyperglycaemic than the non-error group according to several measures (table I). Self monitoring and clinic blood 
glucose concentrations were both slightly (about $2 \mathrm{mmol} / \mathrm{l}$ ) higher, whereas the serum fructosamine concentration was substantially higher in the error group than the non-error group $(2.4(0.4) v 1.8(0.4)$ $\mathrm{mmol} / \mathrm{l} ; \mathrm{p}<0.0001)$. In the error group the raised serum fructosamine concentration (an index of overall glycaemic control) was consistent with the raised clinic blood glucose concentration $(18.9(2.9) \mathrm{mmol} / \mathrm{l})$ but incompatible with the mild hyperglycaemia suggested by self monitoring $(10 \cdot 1(2 \cdot 0) \mathrm{mmol} / \mathrm{l})$. By contrast, in patients in the non-error group, in whom raised clinic blood glucose concentrations $(16 \cdot 8(1 \cdot 6) \mathrm{mmol} / \mathrm{l})$ could not be replicated at home, the serum fructosamine concentration was at the upper limit of the nondiabetic reference range $(<1.8 \mathrm{mmol} / \mathrm{l})$, consistent with the self monitoring data $(8 \cdot 4(1.5) \mathrm{mmol} / \mathrm{l})$.

\section{Discussion}

Doctors managing diabetes commonly face a therapeutic dilemma when a patient's blood glucose concentration at clinic is repeatedly much higher than that recorded by the patient at home. We studied this problem in non-insulin dependent diabetic patients attending our teaching hospital diabetes clinic. Almost half of the patients had significant errors in their monitoring technique despite adequate instruction and retesting. Neither age nor duration of diabetes distinguished this group from those who had correct technique. However, in the error group there was a higher prevalence of physical and cognitive defects that were thought to be sufficient to cause errors in technique. This raises the question, were such problems present when the technique was first taught or did they develop subsequently, an issue which was not addressed in our study. More detailed assessment of the patients' abilities before teaching self monitoring might have prevented some of the errors in technique that we detected. Though visual interpretation of the testing strips was a source of error in patients not using a meter, four patients using a reflectance meter also made errors.

Nineteen $(56 \%)$ patients had no demonstrable error in monitoring technique to account for the glycaemic discrepancy. In these patients, glucose concentrations in both venous and finger prick capillary blood samples were significantly lower than at the clinic visit. This suggests that some aspect(s) of the clinic visit accounted for the atypically high blood glucose concentrations and is analogous to "white-coat hypertension." ${ }^{3}$ Interestingly, white coat hypertension is reported to be significantly more common in diabetic patients. ${ }^{8}$ Psychological stress raises blood glucose concentrations in patients with non-insulin dependent diabetes. ${ }^{9}$ Patients' records showed no evidence of any patient having a severe underlying emotional disorder. The stress of the venepuncture procedure itself was not the main cause of the discrepancy as the home venepuncture glucose estimation was substantially lower than that in the clinic. However, postprandial glucose concentrations should be lower in venous than capillary blood because glucose is extracted from the capillary bed by forearm muscles. We found that home venous blood glucose was slightly, but nonsignificantly, higher than the capillary concentration, which suggests that venepuncture may produce a small glycaemic effect. The overall contribution of stress to the glycaemic discrepancy requires further study.

Our results indicate that retesting of self monitoring technique and measurement of glycated proteins will help assess the cause(s) of discrepancies between clinic and self recorded blood glucose concentrations. Measurement of glycated proteins will provide an objective index of overall glycaemic control. A value consistent with self monitoring data would imply clinic related transient hyperglycaemia, whereas a value consistent with the raised clinic blood glucose concentration would suggest incorrect self monitoring values. Though our results suggest that the inaccurate self recorded values are usually due to errors in technique, occasionally patients may falsify data. We have found, however, that such falsification is uncommon in patients with non-insulin dependent diabetes. ${ }^{10}$ Unfortunately, such therapeutic decision making in diabetes clinics is usually hindered by unavailability of the glycated protein concentration at the time of the clinic visit. A rapid-assay system for measuring glycated proteins needs to be developed.

As a result of this study we have altered some aspects of our diabetes management. Patients are now assessed more carefully before instruction in self monitoring. For patients with cognitive defects or physical disability that can make monitoring inaccurate another person, such as a family member or district nurse, is asked to assist with or perform the tests. Monitoring technique is retested at regular intervals (every one to two years) as a routine, with retraining or transfer of the task when necessary.

We are grateful for the help of Sisters C Carrington and M Jeffries.

1 Consensus statement on self-monitoring of blood glucose. Diabetes Care 1987;10:95-9.

2 McLay J, Ashwell S, Carrington C, Borkman M, Campbell L. Characterisation of outpatient population in a large urban outpatient clinic. Proceeding of the Australian Diabetes Educators Conference 1987: E3.

3 Long J, Gillilan R, Lee SG, Kim CR, Plantholt S. White-coat hypertension: detection and evaluation. Md Med f 1990;39:555-9.

4 Molnar GD, Taylor WF, Langworthy A. On measuring the adequacy of diabetes regulation: comparison of continuously monitored blood glucose patterns with values at selected time points. Diabetologia 1974;10:139-43.

5 Rose RM, Sachar E. Endocrine responses to stress. In: Williams RH, ed. Textbook of endocrinology. Philadelphia: Saunders, 1981:654-5.

6 Fell LR, Shutt DA, Bentley CJ. Development of a salivary cortisol method for detecting changes in plasma "free" cortisol arising from acute stress in sheep. Aust Vet $\mathcal{F}$ 1985;62:403-6.

7 Schleicher ED, Vogt BW. Standardisation of serum fructosamine assays. Clin Chem 1990;36:136-9.

8 Burgess $\mathrm{E}$, Mather K, Ross S, Josefsberg Z. Office hypertension in type 2 (non-insulin-dependent) diabetic patients. Diabetologia 1991;34:684.

9 Surwit RS, Feinglos MN. The effects of relaxation on glucose tolerance in noninsulin-dependent diabetes. Diabetes Care 1983;6:176-9.

10 Campbell LV, Reinhardt J, Ashwell S, McLay J. Verification of blood glucose recording during outpatient stabilisation of diabetes. Diabetes 1991;40:140.

(Accepted 11 September 1992) 